\title{
Myocarditis complicating ethylene glycol poisoning in the absence of neurological features
}

\author{
D.W. Denning, ${ }^{1}$ A. Berendt,${ }^{1}$ Y. Chia,${ }^{2}$ and S.H. Morgan ${ }^{3}$
}

Departments of ${ }^{1}$ Infectious Diseases (Lister Unit), ${ }^{2}$ Histopathology and ${ }^{3}$ the Division of Inherited Metabolic Diseases, Northwick Park Hospital and Clinical Research Centre, Watford Road, Harrow, Middlesex HAl $3 U J, U K$.

\begin{abstract}
Summary: The consequences of ethylene glycol intoxication are described in a 42 year old man who presented with a profound metabolic acidosis, cardiogenic shock and renal failure. The clinical and electrocardiographic findings suggested a myocarditis and this was confirmed by endomyocardial biopsy. The striking absence of neurological features in this patient delayed diagnosis, which was not suspected until calcium oxalate crystals were demonstrated at renal biopsy. Retrospective analysis of serum retained at his admission to hospital confirmed toxic levels of ethylene glycol. Following a period of intensive 'cardio-respiratory support and peritoneal dialysis he made a good recovery with cardiac and renal function returning to normal.
\end{abstract}

\section{Introduction}

Ethylene glycol is a sweet-tasting liquid which is commonly incorporated into the formulation of radiator 'anti-freeze' agents. A total dose of $100 \mathrm{~g}$ is usually fatal in adults if untreated, but the consequences of intoxication are rarely seen in clinical practice, less than 50 cases being reported to the Guy's Poisons Unit annually (J. Henry, personal communication). The classical presentation is of convulsions and focal neurological abnormalities culminating in coma followed by the development of profound metabolic acidosis, cardio-respiratory failure and oliguric renal failure.

We report an unusual case of ethylene glycol poisoning with cardiac and renal failure in the absence of neurological features. Recovery of myocardial function took more than 3 months. The patient denied self administration of any poisons or household chemicals.

Case history

A 42 year old Asian man was admitted acutely

Correspondence: S. H. Morgan, M.B., M.R.C.P., South West Thames Regional Renal Unit, St Helier Hospital, Carshalton, Surrey SM5 1AA

Accepted: 16 June 1988 unwell with a 4-day history of chest pain, breathlessness and productive cough. On examination he was dehydrated, breathless at rest and had a fever. There was peripheral cyanosis and he had cold extremities. He had a sinus tachycardia, and was hypotensive (blood pressure $90 / 40 \mathrm{~mm} \mathrm{Hg}$ ). The heart sounds were normal and there were bilateral coarse crackles at the lung bases.

Investigations showed a haemoglobin of $9.8 \mathrm{~g} / \mathrm{dl}$, white cell count $8.2 \times 10^{9} / 1$ with a neutrophilia, platelets $179,000 \times 10^{9} / 1$ and ESR $26 \mathrm{~mm} / \mathrm{h}$. The prothrombin time and KCCT were prolonged. Urea was elevated at $14.7 \mathrm{mmol} / \mathrm{l}$, with a serum creatinine of $205 \mu \mathrm{mol} / 1$. The electrolytes were normal, and the creatine kinase was 1294 IU (normal range 20 175 IU) with CK-MB fraction $<1 \%$. The chest Xray showed changes suggestive of pulmonary oedema and an electrocardiogram (ECG) showed sinus tachycardia, rate $150 / \mathrm{min}$, with inverted $\mathrm{T}$ waves in V1-V3 and T-wave flattening in the lateral leads.

The patient was transferred to the intensive therapy unit with a presumptive diagnosis of myocarditis. He had a metabolic acidosis with a $\mathrm{pH}$ of 7.01 and bicarbonate of $6.0 \mathrm{mmol} / 1$. The serum lactate was $13.2 \mathrm{mmol} / 1$ (normal range 1.0 $1.8 \mathrm{mmol} / \mathrm{l})$. The cardiac output was measured as 2.6 litres/min and the pulmonary artery wedge pres-

(C) The Fellowship of Postgraduate Medicine, 1988 
sure was elevated. He was treated with dopamine, dobutamine and adrenaline and methylprednisolone. A basal pneumonia developed, and because of profound hypoxaemia $\left(\mathrm{PaO}_{2}<5 \mathrm{kPa}\right)$, required assisted ventilation for 10 days. Respiratory function improved with antibiotic therapy, but because of unexplained episodes of apnoea, the endotracheal tube was left in situ. These resolved after 5 days.

Rapidly progressive renal failure developed and peritoneal dialysis was commenced 4 days after admission when the blood urea had risen to $54.8 \mathrm{mmol} / 1$. There was a dramatic rise in creatine kinase with a maximum value of $14,000 \mathrm{IU}$ on the 3 sixth hospital day. The CK-MB fraction remained $\stackrel{\mathbb{Q}}{\square}$ $<1 \%$. He developed dry gangrene of his fingers $\leftarrow$ and toes, attributed to a combination of prolonged $\overrightarrow{\vec{D}}$ hypotension and adrenaline-induced vasoconstriction.

Endomyocardial biopsy showed focal muscle cell necrosis with a diffuse infiltrate of mononuclear cells. The features were in keeping with myocarditis (Figure 1).

The renal biopsy contained 16 glomeruli, one of which was sclerosed. The remainder were of normal $\vec{\circ}$ appearance. Approximately one third of the tubules showed dilatation and flattening of the epithelium,

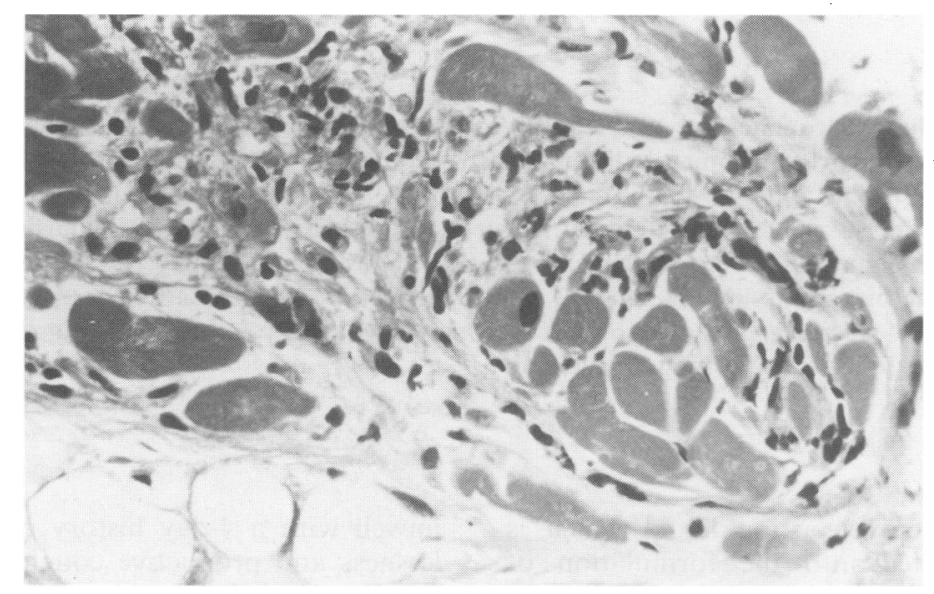

Figure 1 Cardiac muscle biopsy. There is focal cell necrosis with surrounding mononuclear cells $(\times 362$ magnification).

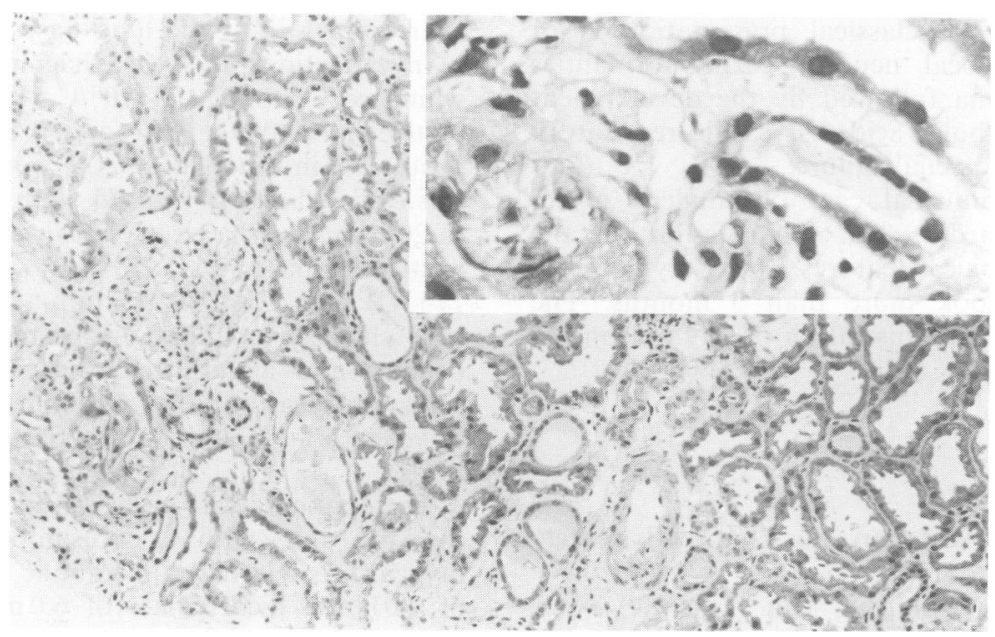

Figure 2 Renal biopsy. Tubules showing changes of acute tubular necrosis. An occasional birefringent crystal is present in the tubule, see inset. ( $\times 94.2$ magnification). 
with occasional mitotic figures, and hyaline casts. There was occasional birefringent material in the tubules. In the interstitium there was mild focal oedema and small collections of mononuclear cells. The features were those of acute tubular necrosis with oxalate crystals in the tubules (Figure 2).

Blood from the time of admission was therefore retrospectively analysed for toxins and showed a level of ethylene glycol of $0.4 \mathrm{~g} / \mathrm{l}$. The patient and his family denied any knowledge of ingestion of antifreeze or any ethylene glycol containing substance. There were, however, considerable medical and social problems in the family.

The patient returned to the general ward 29 days after admission. A multiple gated pool scan (MUGA) was performed which showed an ejection fraction of $24 \%$. A follow-up MUGA scan two months later showed a rise in the ejection fraction to near normal at $46 \%$. He remains well apart from ischaemic pain in his finger and toe tips.

\section{Discussion}

Since the first description of ethylene glycol poison-

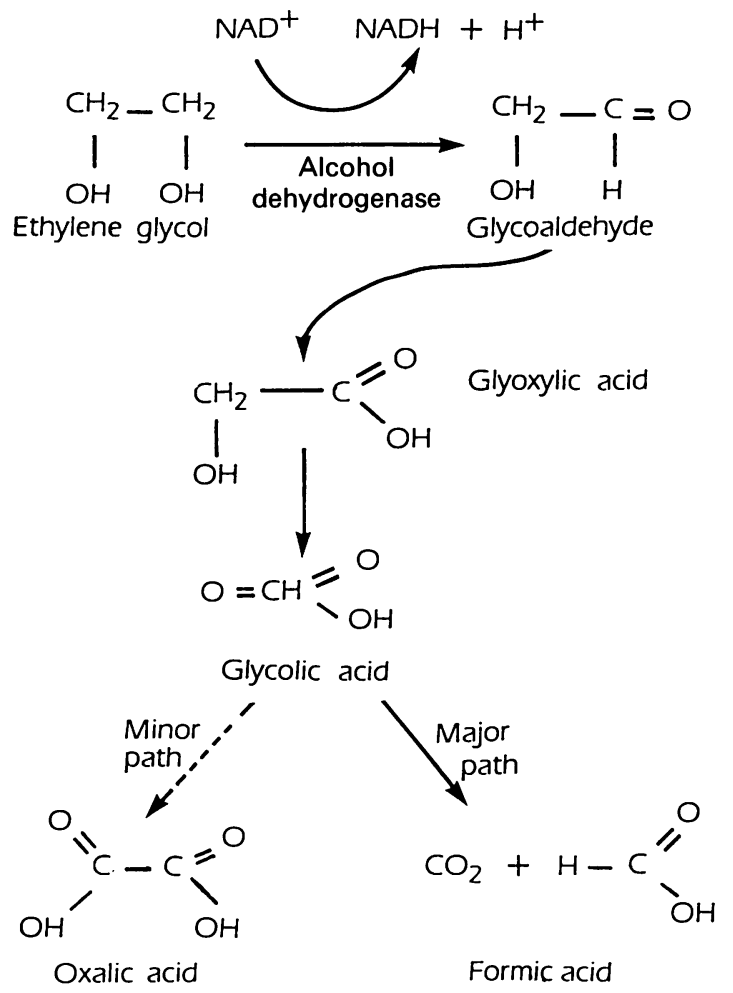

Figure 3 Metabolism of ethylene glycol. ing in $1946,{ }^{1}$ it has become evident that the clinical syndrome manifests in three fairly distinct stages ${ }^{2}$ related to the timing of the appearance of different metabolites of ethylene glycol (Figure 3). The initial stage is characterized by neurological features and is related to high concentrations of glycoaldehyde. Symptoms usually occur within 12 hours of ingestion. Within 24 hours high concentrations of glyoxylate and oxalate result in a profound metabolic acidosis, characterized biochemically by expansion of the 'anion gap', and clinically by cardiorespiratory failure. If the patient survives, renal failure subsequently develops due to a combination of hypotension and the direct toxicity of oxalic acid and other metabolites. Oxalate crystalluria and deposition of calcium oxalate in the kidney, and other tissues may be marked. ${ }^{3}$ It is thought that the minimal fatal dose in an adult is about $100 \mathrm{ml}$.

Although the presentation in our patient was unusual, with a striking absence of neurological signs, the clinical features, lactic acidosis and renal parenchymal deposition of calcium oxalate, taken in the context of a raised blood ethylene glycol level measured in retrospect, support the diagnosis.

ECG abnormalities have been neglected in previous case reports, although wide complexes and flat $T$ waves with no intrinsic atrial activity are described. ${ }^{4}$ Descriptions of pathological changes in the heart at autopsy are also sparse, and were not even recorded in one large series. ${ }^{1}$ In two other series, where the cardiovascular system was examined in detail in 5 patients at autopsy, the findings were generally of petecheal haemorrhage and cardiac dilatation with degenerative myocardial changes 5.6 ; an inflammatory cell infiltrate was not found. Our initial clinical diagnosis of a myocarditis was supported by the findings on endomyocardial biopsy. The features were, however, nonspecific, and would not differentiate between viral myocarditis and the toxic myocarditis reported with a wide range of drugs and chemicals. ${ }^{7}$ The glycoaldehyde metabolites of ethylene glycol have profound cellular effects, inhibiting oxidative phosphorylation, glucose metabolism and protein synthesis as well as the mechanisms of DNA repair, ${ }^{1}$ and the recovery of myocardial function seen in this patient is surprising. The residual degree of mild impairment documented isotopically may reflect 'long' standing structural damage, the initial severity of the cardiac failure was due to local biochemical effects on myocardial contractility including acidosis and hypocalcaemia due to calcium oxalate deposition.

Had a diagnosis of ethylene glycol poisoning been suspected earlier and confirmed biochemically, the prompt infusion of ethanol would have been indicated. In this atypical case, there is no doubt 
that survival was influenced by early peritoneal dialysis both removing ethylene glycol metabolites, and supporting renal function.

\section{Acknowledgements}

We are grateful to Dr Hillas Smith for encouraging us in

\section{References}

1. Pons, C.A. \& Custer, R.P. Acute ethylene glycol poisoning. A clinicopathologic report of eighteen fatal cases. Am J Med Sci 1946, 211: 544-552.

2. Parry, M.F. \& Wallach, R. Ethylene glycol poisoning. Am J Med 1974, 57: 143-150.

3. Turk, J., Morrel, L. \& Avioli, L.V. Ethylene glycol intoxication. Arch Int Med 1986, 146: 1601-1603.

4. Scully, R.E., Galdabini, J.J. \& McNelly, B.U. Case records of the Massachusetts General Hospital (Case 38). $N$ Engl Med J 1979, 301: 650-657. reporting this case, and in particular to Dr John Henry at the Poisons Unit, Guy's Hospital for the ethylene glycol $\stackrel{\mathbb{Q}}{\stackrel{2}{2}}$ assays.

5. Khan, H.S. A recovery from ethylene glycol poisoning: A case of survival and two fatalities from ethylene glycol including autopsy findings. Ann Int Med 1950, 32: 284-289.

6. Haegmann, P.O. \& Chiffelle, T.R. Ethylene glycol poisoning, J Lab Clin Med 1948, 33: 573-584.

7. Aretz, H.T. Diagnosis of myocarditis by endomyocardial biopsy. Med Clin N Am 1986, 70: 1215-1226. 